\title{
Environment Factors Monitoring System Based on CAN bus
}

\author{
http://dx.doi.org/10.3991/ijoe.v12i05.5722 \\ Shihong Li, Sasa Zhu and Yan Jin \\ Wenzhou Vocational College of Science and Technology, Zhejiang, China
}

\begin{abstract}
In order to solve the greenhouse environmental factors monitoring system wiring is difficult, high cost, single parameter monitoring, lack of flexibility and scalability, which can't meet the requirements of the modern intelligent greenhouse. In this study, a new remote monitoring system was established. In terms of the hardware, the SCM C8051F040 was adopted to collect the environmental parameters such as temperature, humidity, light intensity, $\mathrm{CO}^{2}$ concentration and so on. All data were uploaded via CAN bus and GPRS to the remote host computer.The collected data would be sent to the relevant agricultural management personnel in real time through the GPRS network so that the management could be carried out. Based on Kingview software platform, which was developed to use graphic language and command language system of the PC. Preliminary experimental results show that the system has the correct data transmission, stable operation, good real-time performance and accurately monitor the environmental factors in the greenhouse. The system has the advantages of flexible networking and strong expansibility and intuitive interface, which can well meet the needs of environmental factors monitoring in greenhouse..
\end{abstract}

Index Terms-Greenhouse, Monitor ,CAN bus, GPRS, KingView

\section{INTRODUCTION}

Environmental factors such as temperature, humidity, light intensity, concentration of $\mathrm{CO}^{2}$, etc. are closely related to the growth, development and yield of crops. The requirements of different crops for different environmental factors vary a lot in the course of their growth, and the key environmental factors to the same crop are also dissimilar in different growth periods. With the development of science and technology, modern techniques and methods such as computer technology, automation technology, communications technology, etc. have been used to design a remote monitoring system. Monitoring various environmental factors for crop growth, as well as analyzing and processing the data obtained, is crucial to improve crop quality and yields, which could not only enhance the management of agriculture, but also facilitate the modernization of agriculture.

\section{StATE Of ThE ART}

Related research conducted by scholars at home and abroad include, for instance, Lichang Li et al. [1]designed a greenhouse wireless monitoring system based on ZigBee and GPRS networks to monitor the temperature, humidity, concentration of $\mathrm{CO}^{2}, \mathrm{pH}$ value of soil, light intensity and other parameters within the greenhouse; Hengjun Zhu et al. [2]built a CAN bus-based greenhouse temperature monitoring system using CAN bus technology and the temperature sensor DS18B20 with single-chip microcomputers (SCM) as the core; To automatically adjust the environmental factors in greenhouses for crop growth, Gaofeng Peng[3] designed an intelligent automatic greenhouse environment measurement and control system by means of CAN bus transmission; In combination with temperature and humidity sensors as well as GPRS modules, Zhiqiang Wang et al. [4]designed a GPRS technology-based wireless granary temperature and humidity monitoring system with SCM as its core according to the feature that the environmental monitoring points for largesized grain depots are scattered; In 2010, Pengzhan Chen and Baifen Liu[5] designed, by virtue of CAN technology, an intelligent greenhouse control system that could intelligently regulate the temperature and humidity within the greenhouse on the basis of the preset boundary values of air temperature and humidity; Antonio-Javier GarciaSanchez[6], J.A. LópezRiquelme et al.[7] realized environmental parameter and video monitoring based on the wireless sensor network (WSN), but the facilities of this environmental monitoring system did not attach due significance to the low power consumption of the wireless sensor nodes; And the plant physiology and environmental monitoring system "PTM-48M" created by PhyTech, a Israeli company, which could monitor the soil and air environment in real time are mainly used for long-term monitoring of the plant physiology in the laboratory[8].

At present, China's greenhouse monitoring systems are generally designed for monitoring the environmental factors of large-scale and industrialized greenhouses, therefore with high costs. However, to those small-sized greenhouse owners, such as with only a dozen or even less than ten greenhouses, they can't afford a full set of greenhouse monitoring system, which hinders the popularity of greenhouse monitoring systems. Meanwhile, it also happens that some greenhouse monitoring systems could only monitor one rather than several greenhouses simultaneously[9]. Moreover, to import large-scale multi-functional monitoring systems is not only expensive, but also inapplicable to the condition of our country due to different climatic conditions [10].

Taking above-mentioned problems into account, this paper presents a method that integrates CAN bus, GPRS and Kingview Programmable software technology for designing an intelligent greenhouse monitoring system. Such a system could not only monitor the environmental factors' parameters within the greenhouse in real time, such as temperature, humidity, light intensity, $\mathrm{CO}^{2}$ concentration, etc., but also realize the adjustment of tempera- 
ture, humidity, ventilation, light intensity, etc. through related control modules. Also, it could send alarms over environmental factors to the administrator in time in the form of short messages via GPRS networks to facilitate the timely processing of the information. The remainder of this paper is organized as follows. Section 3 describes the overall design of the system block diagram, the hardware structure of the system, the choice of each device, the system of the slave computer and PC software. Sections 4 presented a real experimental verification. Conclusions are summarized in Section 5.

\section{Methodology}

\section{A. The Overall Design of the System}

The overall structure diagram of the entire system is shown in Figure. 1. The system is made up of three parts including a monitoring center, a GPRS module and a data collection terminal, the latter of which is composed of an environmental factor detection module, a control module, a CAN bus interface module and a CPU. Taking microcontroller chip C8051F040 as the core, the data collection terminal is responsible for collecting environmental factors within the greenhouse and realizing CAN communications among greenhouses and GPRS communications. Inside the chip, there is a controller area network (CAN) controller and CAN protocol is used to achieve serial communications. This CAN controller is in accordance with both Bosch Norm 2.0A (basic CAN) and 2.0B (fullfeatured CAN), which facilitates CAN network communication design [11]. The environmental factor detection module is made up of a temperature and humidity detection module, a light intensity detection module and a $\mathrm{CO}^{2}$ concentration detection module. The control module of each function is mainly used to achieve temperature, humidity, ventilation and light intensity adjustment within the greenhouse to ensure that dehumidification, cooling, ventilation, supplementary light and other equipment could work properly.

The CAN bus interface module is responsible for CAN protocol conversion and bus driving. The GPRS module is responsible for data switching between each greenhouse and the host computer monitoring center [12]. And the monitoring center will get sampled data via the GPRS network and then complete data storage, processing and other operations.

\section{B. Connection of Each Sensor with SCM within the Data Collection Terminal}

The temperature and humidity detection module adopts the integrated single-chip digital humiture sensor SHT10 (Sensirion, Switzerland) that uses CMOS process micromachining patent technology to ensure extremely high reliability and excellent stability. The sensor consists of a capacitive polymer humidity measuring element and a bandgap temperature measurement component, and it is seamlessly integrated into SCM with a 14-bit A/D converter and a 2 -wire digital interface, making the product be characterized by the advantages such as low power consumption, fast response, great anti-interference ability, etc. [13].

The input pin of SCK (serial clock) is the synchronous clock of MCU and SHT10. The serial data pin of DATA is used for data transfer between MCU and SHT10. The status of DATA changes after the falling edge of SCK and is valid at the rising edge of SCK. During data transfer, the data wire of DATA must remain stable when SCK is at a high level. To avoid data contention, a pull-up resistor is externally connected to DATA to pull up the signal to a high level [14].

Currently, three kinds of $\mathrm{CO}^{2}$ sensors are widely used, including infrared ones, electrochemical ones and heat conduction ones. What the $\mathrm{CO}^{2}$ concentration detection module of this system uses is the infrared $\mathrm{CO}^{2}$ gas module T6004. The output signal of this sensor could be either digital output or analog output, the latter of which is utilized in this paper to simplify the system design. The ana$\log$ output of T6004 is $0-4 \mathrm{VDC}$, after attenuated by the resistor divider, it is connected to the $A / D$ conversion input channel of C8051F040 [15].The circuit of the temperature, humidity and $\mathrm{CO}^{2}$ concentration detection modules are shown in Figure. 2.

The main function of the light intensity sensor module is the real-time and accurate acquisition of the light intensity in the greenhouse environment, and the data obtained will be then transferred in time to C8051F040. Light intensity sensors could be photosensitive resistance, photodiodes, phototransistors, photovoltaic cells, etc. Photovoltaic cells are characterized by many advantages such as stable performance, excellent frequency characteristics, long service life, high temperature resistance, etc. [16], so what the system adopts in this study is the silicon photovoltaic cell BPW34S. The output voltage signal of the photosensitive probe will be amplified through the op amp to get the $0-2 \mathrm{~V}$ voltage signal which will be then transferred to the A/D conversion channel of C8051F040 to obtain digital signals [17].

The CAN bus interface module is mainly designed to realize CAN protocol conversion and bus driving. CAN bus is a serial communication network that could effect-

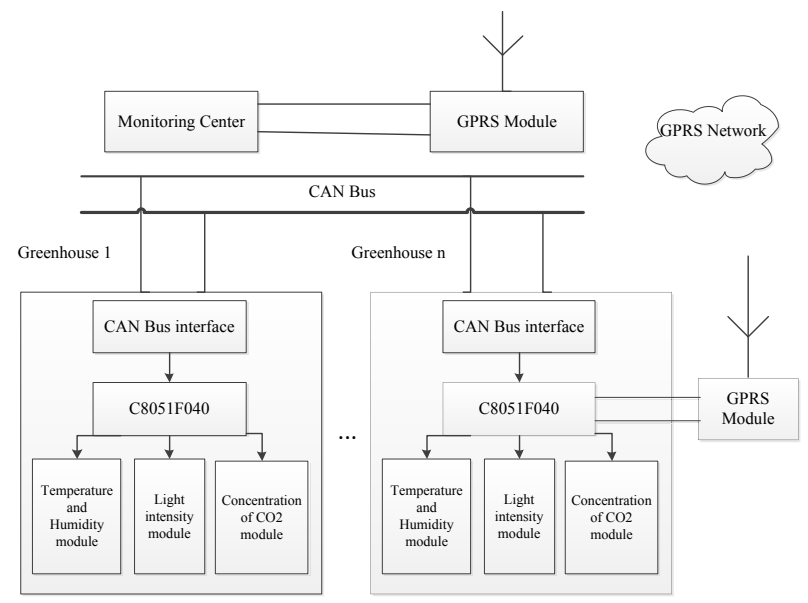

Figure 1. System diagram

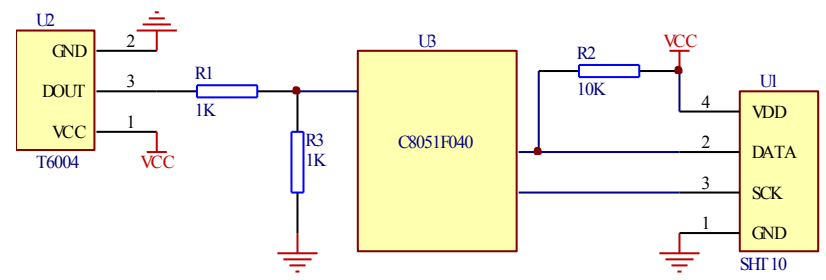

Figure 2. Module of temperature, humidity and $\mathrm{CO}^{2}$ concentration 
tively support both distributed and real-time control. The monitoring module of each greenhouse forms a local areanetwork (LAN) through CAN bus. All modules on the CAN bus could send information to the other modules in the network at any time on its own initiative regardless of primary from secondary to realize free communications between modules. As C8051F040 is internally integrated with a CAN bus controller, an external CAN transceiver is enough. In this system, the CAN transceiver is P82C250 purchased from Philips. P82C250 is an interface between the CAN bus controller and the physical bus, and it is a standard high-speed CAN transceiver responsible for interconverting the digital signal of the controller with the differential signal of the CAN bus [18]. To enhance the anti-interference ability, a photoelectric isolate circuit is set between the CAN bus controller and the transceiver using the high-speed optocoupler 6N137 [19].

\section{Connection of SCM with the GPRS Module}

After market research, the wireless GPRS module G200 provided by Beijing Jammy Telecom Equipment Co., Ltd. was selected. G200 is a GPRS module embedded with a TCP/IP protocol stack, which is flexible, reliable and convenient to use. Its users do not have to worry about the RF circuit, related hardware connection or AT instruction sets of the module [20]. Since the serial port of C8051F040 is the standard TTL level, while that of the GPRS module is the standard RS-232C protocol level (logic " 1 " is from $-3 \mathrm{~V}$ to $-15 \mathrm{~V}$; logic " 0 " is from $3 \mathrm{~V}$ to $15 \mathrm{~V}$; while any voltages between $-3 \mathrm{~V}$ to $+3 \mathrm{~V}$ are in an undefined logic state and the voltage lower than $-15 \mathrm{~V}$ or higher than $+15 \mathrm{~V}$ is also considered insignificant;) the level must be between $\pm(3-15) \mathrm{V}$ at work [21]. To achieve serial communications between the two modules, this system adopts the MAX3232E chip to provide the system with the level shifting function. A standard 9-pin port is installed, but three cables are enough in actual use.

\section{Slave Computer Software Design}

Using the modular C language programming method, this system is featured by good portability which indicates that the system could be expanded or trimmed according to users' actual needs. The software mainly covers the data collection module, the CAN communication module and the GPRS communication module.

The sensor data acquisition and processing module is primarily responsible for collecting some analog quantity and digital quantity, of which the former mainly includes $\mathrm{CO}^{2}$ concentration and light intensity while the latter mainly refers to temperature and humidity. The analog signal could be precisely measured because C8051F040 of the system is embedded with a 12-bit A/D converter. If the data collected by the system exceed the preset ones, it will alarm and start corresponding control devices. Otherwise, the data collected will be sent to the CAN bus.

\section{E. CAN Communication Module}

After the program is started, CAN communication ports will be firstly conFigureured to initialize CAN, complete the setting of the CAN control register and the bit timing register, as well as initialize message sending and receiving objects. Based on the priority level, it will judge whether it has already received data or not. If the answer is positive, it will start to read and process the data in the receiving buffer zone. Afterwards, it will determine if there are any requests for data transmission. If there are, then the data will be sent to the sending buffer zone and data transmission is started.

CAN message sending and receiving is automatically carried out by the CAN controller. Users only need to transfer corresponding data to the sending buffer zone according to the identifier in the remote frame, input the encoded message into the command, and then start sending. The same with message sending, message receiving program only needs to read and process the data in the receiving buffer zone. The basic method is consistent with that of the sending program [22]. Part of the code of the sending program is as follows:

$$
\begin{aligned}
& \text { void CAN_TR (char Msg_Num) } \\
& \text { SFRPAGE = CAN0_PAGE; } \\
& \text { CAN0ADR = IF1DATAI; } \\
& \text { CAN0DAT = TXbuffer; } \\
& \text { CAN0ADR = IF1CMDRQST; } \\
& \text { CAN0DATL = MsgNum; }
\end{aligned}
$$$$
\text { \} }
$$

\section{F. GPRS Module Parameter Setting}

As an important part of the system's remote network, the GPRS module plays a crucial role in data transmission of the data packet from underlying data to the host computer in the entire monitoring system. The parameters of the G200-type GPRS module should be set in accordance with different application requirements before it is used [23]. It could be conducted by running CQ11T0100-G200-type GPRS Module Testing and Setup Software on computer. The parameters that need to be set mainly include the module's ID address, destination address, operation mode, serial data format, baud rate, etc. Specific parameters are set as follows: The ID address of the data sending terminal module is " 01 " and of the data receiving module is " 00 "; the project number of both modules is " 0 "; the operation mode is active; the communication mode is transparent transmission; and the baud rate is $9,600 \mathrm{bps}$.

\section{G. Design of PC Software}

The host computer monitoring program is achieved by the Kingview Programmable software. The monitoring system software developed by means of Kingview is a new type of industrial automatic control system that could make the best of graphic editing features of Windows to generate monitoring pictures conveniently. Furthermore, the state of the control equipment is displayed animatedly. With an alarm window, real-time trend curves, etc., it is easy and convenient to get various reports [24]. The monitor screen is shown in Figure. 3. It could display the real-time data of each parameter measured in the greenhouse, the state of GPRS data, and whether each datum alarms, which is conducive to visually learning about the latest information of the greenhouse. In addition, it is also equipped with control buttons, through which the growth environment of each greenhouse could be remotely controlled. What's more, it could also provide powerful functions such as reporting, data curve analyzing, etc. to facilitate the analysis and summary of historical data [25]. 


\section{RESUlt ANALYSIS AND DISCUSSION}

The environmental factors of a vegetable greenhouse of our institution were monitored using this system, and the data obtained was shown in Figure 4 and Figure 5. The temperature and humidity curves obtained were basically in line with the actual changes in temperature and humidity. However, with the constant increase in temperature and light intensity, the concentration of $\mathrm{CO}^{2}$ declined. In the case of abnormal temperature and humidity, the system could send audible and visual alarm signals and at the same time, activate corresponding control devices. The experiment indicates that the system can work properly and efficiently, and the control command of the host computer and the data of each greenhouse can be accurately transmitted and exchanged at a high speed.

\section{CONCLUSION}

To be able to solve complex wiring, high construction costs, cutting the current node inconvenient problem exists greenhouse environmental monitoring system, we used CAN bus, GPRS and conFigureuration software technology designed for reliable operation and complete remote monitoring convenient and efficient monitoring system of intelligent greenhouses . This system could upload the data modules collected via the CAN bus and monitor the working state of the greenhouse nodes in real time though the host computer. Meanwhile, by cutting down the number of bus nodes, it could adjust the number of greenhouses conveniently without influencing the stability of the entire system. Additionally, through making the best of existing GPRS networks, it had dramatically reduced the construction cost. More than that, it could also receive temperature and humidity alarms conveniently via the GPRS network to ensure timely processing. With such a system, greenhouse-related people could grasp the latest information about their greenhouses, which was crucial for them to learn about and better the growth environment of the greenhouses and improve crop yields.

(1) Greenhouses more perfect solution was proposed in this paper, the greenhouse environmental factors in the use of a variety of different sensor through the real-time collection of C8051F040 MCU, upload the data through CAN bus, according to the orders of the PC for greenhouse monitoring and management, also through the GPRS network, the data information was sent to the agricultural management.

(2) The paper introduces the GPRS technology had a packet-switched mode, low cost, increasing SMS alarm function, so that agriculture could grasp the greenhouse environment information and better management and operation.

(3) By make full use of the advantages of CAN bus, through the CAN bus distributed control system, real-time of the greenhouse environmental factors upload, transmission distance farther, strong anti-jamming capability ,low cost and other advantages.

(4) PC monitoring platform was developed based on Kingview, we achieved the greenhouse environment factor acquisition, real-time data display, alarm and online access and other functions.

In summary, in this paper, the greenhouse environment factor of the data transmission and real-time monitoring and so on had carried on the thorough research and explo-

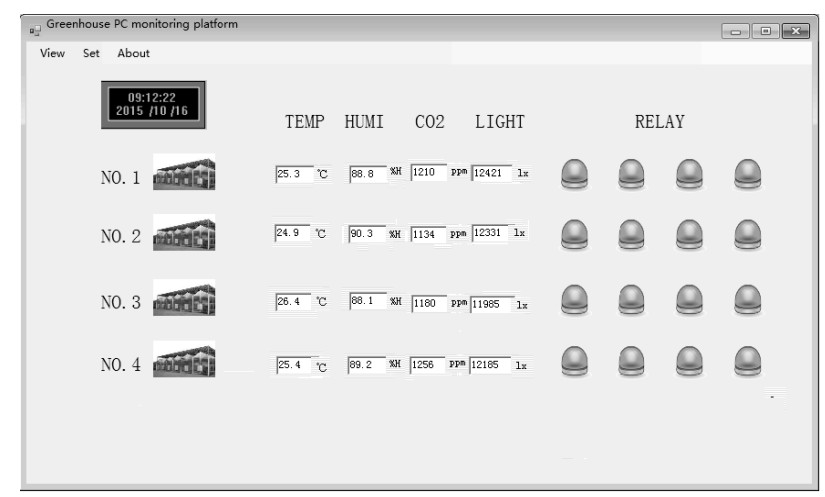

Figure 3. Monitor screen

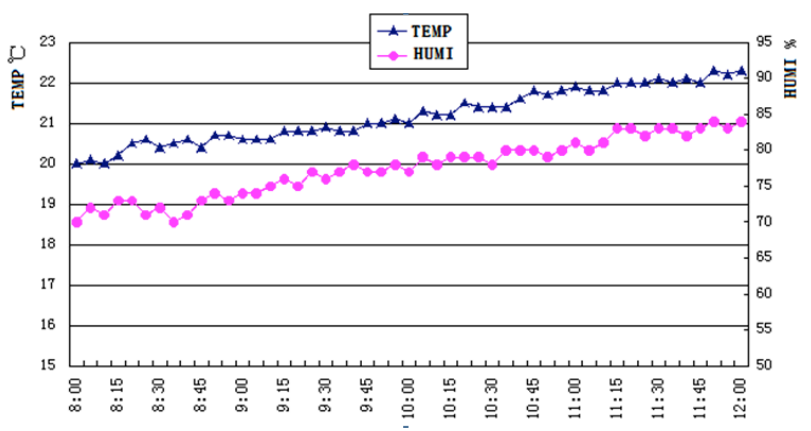

Figure 4. Monitoring curve of temperature and humidity

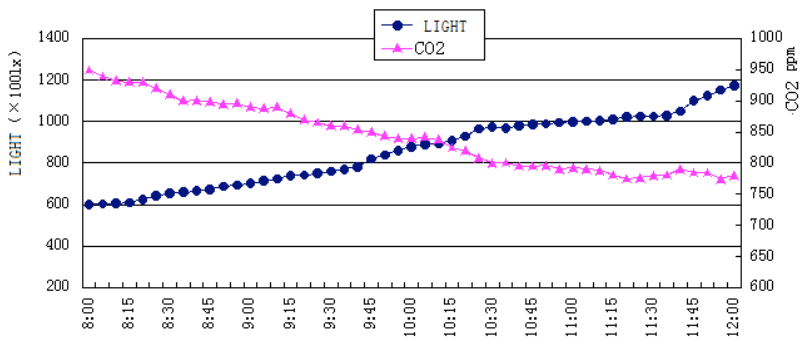

Figure 5. Monitoring curve of $\mathrm{CO}^{2}$ and light intensity

ration and the basic functions of this system was verified by experiment.

In the process of the development of this system, there are some shortages and areas for improvement. In this paper, the environmental factors of monitoring only air temperature, humidity, $\mathrm{CO}^{2}$ concentration, light and other environmental factors such as soil moisture, soil nutrient parameters such as have not monitoring, in practical work, you can be of different parameters depending on the needs of crops monitor. This system can't across regions, large area monitoring. The PC function and interface of system needs to be improved. However, there are still many aspects worth further exploring in terms of how to ensure the proper and stable running of each module due to the complex environment of the greenhouse.

\section{REFERENCES}

[1] Li Liyang, Wang Huabin and Bai Fengshan, "Design of Wireless Monitor System of Greenhouse Based on ZigBee and GPRS", Computer Measurement \& Control, vol.20,no.2, ,pp. 3148-3150, 2012.

[2] Zhu Hengjun, Yu Hongbo and Wang Fazhi, "Measurement and Control System for Temperature in the Greenhouse Base on CAN Bus", Microelectronics \& Computer, vol.29,no.5,pp. 183-187, 2012. 
PAPER

ENVIRONMENT FACTORS MONITORING SYSTEM BASED ON CAN BUS

[3] Peng Gaofeng, "Intelligent Automatic Measurement and Conditioning System Research on Greenhouse Environment", Computer Measurement \& Control,vol.20,no.10,,pp. 2664-2665, 2012.

[4] Wang Zhiqiang and Chen Ping, "Design of Temperature and Humidity Monitoring System of Grain Depot Based on GPRS Technology”, Anhui Afri. Sci. vol.38,no.14,,pp. 7552-7553, 2012.

[5] Chen, Pengzhan, Liu. Construction of Intelligent Greenhouse Control System Based on CAN bus.

[6] Antonio-Javier Garcia-Sanchez, Felipe Garcia-Sanchez and Joan Garcia-Haro, "Wireless sensor network deployment for integrating video-surveillance and data-monitoring in precision agriculture over distributed crops". Computers and Electronics in Agriculture, no.2, pp. 288-303, 2011. http://dx.doi.org/10.1016/j.compag. 2010.12.005

[7] J A López Riquelme, Soto F and Suardíaz J, "Wireless Sensor Networks for precision horticulture in Southern Spain". Computers and Electronics in Agriculture, no.1, pp. 25-35, 2009. http://dx.doi.org/10.1016/j.compag.2009.04.006

[8] Zhang Jie,Li Aicheng and Li Jianlong, "Research of Real-time Image Acquisition System Based on ARM7 for Agricultural Environmental Monitoring”,pp:6216-6220,2011.

[9] Cui JingWei,'Design and research of wireless temperature monitoring system for greenhouse". Taiyuan University of Science and Technology, Taiyuan ,2013.

[10] Zhang Xin,Chen Lansheng and Zhao Jun, "Design and application of intelligent agricultural greenhouse based on Internet of things technology", Chinese Agricultural Mechanization, vol.36, no.5,pp. 90-95, 2015.

[11] He Yanping,He Hui and SongJia, "Design of intelligent node in the CAN bus system based on C8051f040", Microcomputer Information,vol.23 ,no. 32, pp. 63-64,67, 2007.

[12] Zhu Min, "Design temperature and humidity monitoring system of greenhouse based on CAN bus". Automation \& Instrumentation, no.1, pp. 57-60, 2010.

[13] Hu Zengkang,Wang Lulu and Liu Chaoxin, "Design of temperature and humidity monitoring system for indoor". Science \& Technology Information, no,20, pp. 94-94, 2011.

[14] Sensirion.SHT10 Humidity Sensor Datasheet,2007.

[15] Wang Yinling and Sun Tao, "The Design of Greenhouse Environmental Monitoring of Wireless Sensor Network Nod". Agricultural Mechanization Research,vol.33,no.3,,pp. 113-117, 2011.

[16] Zhao Xin,Ou Jian and Zhu Yuyu,"Research and design of measurement and control system on greenhouse based on wireless communication", Northeast Agricultural University,vol.41 no.7,,pp. 128-134, 2010.
[17] Zhang Wei,Yang Jingfa and Yan Qigeng,"Experiment research on the property of silicon solar cell", Experimental Technology and Management.vol.26, no.9, pp. 42-46, 2009.

[18] Philips Semiconductors.PCA82C250 CAN controller interface DataSheet.2000

[19] Deng Deyuan,Wang Chengdong and Miao Qiang, "CAN-bus Based Online Monitoring System for Humidity and Temperature", Instrument Technique and Sensor, no.12, pp.40-42,60, 2012.

[20] GPRS type G200 module product specification.Beijing Jiemai Communication Equipment Co., Ltd.

[21] Yang Xiao, "The Design of Monitor \& Management System for Vehicle Petroleum Transportation based on GPRS Network". Xiamen University, Xiamen,2008.

[22] Jia Weitao,ZhaoHu and Wang Sangen, "Application of C8051F040 in the Greenhouse Environment Monitoring System Based on CAN-Bus". Agricultural Mechanization Research,vol.33, no.5,pp. 202-206, 2011.

[23] Guo Rongxiang and Wei Shihu, "Telemetry and telecontrol system of wells group pumps based on GPRS", Manufacturing Automation.vol.36 ,no.7,pp. 131-133, 2014.

[24] Fan Yajuan, "Kingview ConFigureuration Software in the Silkworm Cultivation". Techniques of Automation and Applications,vol.28,no.12,pp. 119-120,130, 2009.

[25] XuYong, "Kingview user manual”, Wellintech, BeiJing, 2004.

\section{AUTHORS}

Shihong $\mathbf{L i}$ is a lecturer in the College of Wenzhou Vocational College of Science and Technology. Her research interests include embedded Systems and control technology. (e-mail: 107787722@qq.com).

Sasa Zhu is with the College of Wenzhou Vocational College of Science and Technology, Zhejiang, 325006, China. (e-mail:123069291@qq.com).

Yan Jin is with the College of Wenzhou Vocational College of Science and Technology, Zhejiang, 325006, China. (E-mail: 6455557@qq.com

The was supported by the Public welfare science and technology project of Wenzhou city(R20150030),Science and technology project of Zhejiang province (2014C32031)and Science and technology project of Wenzhou city (N20140047). Submitted 05 April 2016. Published as resubmitted by the authors 06 May 2016. 